Research paper

\title{
TSLP signaling blocking alleviates E-cadherin dysfunction of airway epithelium in a HDM-induced asthma model
}

\author{
Yahui $\mathrm{Hu}^{\mathrm{a}, 1}$, Hangming Dong ${ }^{\mathrm{a}, 1}$, Mengchen Zou ${ }^{\mathrm{a}}$, Chaowen Huang ${ }^{\mathrm{a}}$, Lishan Luo ${ }^{\mathrm{a}}$, Changhui Yu ${ }^{\mathrm{a}}$, \\ JiaLong Chen ${ }^{\mathrm{b}}$, Zhefan Xie ${ }^{\mathrm{a}}$, Haijin Zhao ${ }^{\mathrm{a}}$, Yanqing Le ${ }^{\mathrm{a}}$, Fei Zou ${ }^{\mathrm{b}}$, Laiyu Liu ${ }^{\mathrm{a}, *}$, Shaoxi Cai ${ }^{\mathrm{a}, *}$ \\ ${ }^{a}$ Chronic Airways Diseases Laboratory, Department of Respiratory and Critical Care Medicine, Nanfang Hospital, Southern Medical University, Guangzhou 510515, China \\ ${ }^{\mathrm{b}}$ School of Public Health and Tropical Medicine, Southern Medical University, Guangzhou 510515, China
}

\section{A R T I C L E I N F O}

\section{Article history:}

Received 4 November 2016

Revised 8 February 2017

Accepted 18 February 2017

Available online 21 February 2017

\section{Keywords:}

Asthma

E-cadherin

Airway epithelium

TSLP

House dust mite

PI3K/Akt pathway

\begin{abstract}
A B S T R A C T
Recent studies have indicated that Thymic stromal lymphopoietin (TSLP) plays an important role in the prevention and treatment of asthma. However the role of TSLP in dysfunction of airway epithelial adherens junctions E-cadherin in house dust mite (HDM)-induced asthma has not been addressed. We hypothesized that TSLP contributed to HDM-induced E-cadherin dysfunction in asthmatic BALB/c mice and $16 \mathrm{HBE}$ cells. In vivo, a HDM-induced asthma mouse model was set up for 8 weeks. Mice inhaled an anti-TSLP monoclonal antibody $(\mathrm{mAb})$ before HDM. The mice treated with the anti-TSLP mAb ameliorated airway inflammation, the decreasing and aberrant distribution of E-cadherin and $\beta$-catenin as well as phosphorylation(p)-AKT induced by HDM. In vitro, HDM increased the expression of TSLP and E-cadherin dysfunction by PI3K/Akt signaling pathway. The exposure of $16 \mathrm{HBE}$ to TSLP resulted in redistribution of E-cadherin. These results indicate that TSLP may be an important contributor in E-cadherin dysfunction of HDM-induced asthma. TSLP signaling blocking shows a protective effect in mice and that the PI3K/Akt pathway may play a role in this process.
\end{abstract}

(c) 2017 Published by Elsevier Inc.

\section{Introduction}

Asthma is a common respiratory disease caused by chronic exposure to inhaled allergens, like house dust mite (HDM), that affects up to 300 million people worldwide [1]. As a barrier to the external environment, bronchial epithelium is central to the pathogenesis of asthma and plays a role in translating these gene-environment interactions in asthma [2,3]. Airway epithelium connected molecule E-cadherin can mediate immunological function of airway epithelium through homophilic interactions as well as proliferation, differentiation, and the release of growth factors and proinflammatory factor, such as thymic stromal lymphopoietin (TSLP) [4,5]. E-cadherin connects adjacent cells and controls the outcome of the response to allergens through its interaction with Tregs and DCs [5,6].The loss and redistribution of E-cadherin may facilitate the pro-inflammatory activities of the epithelium and contributes significantly to asthma pathogenesis $[4,7]$.

\footnotetext{
* Corresponding authors at: Chronic Airways Diseases Laboratory, Dept of Respiratory and Critical Care Medicine, Nanfang Hospital, Southern Medical University, Shatai Road 1023, Guangzhou 510515, China.

E-mail address: caishaox@fimmu.com (S. Cai).

1 These authors made equal contributions to this paper.
}

TSLP is a member of the 4-helix-bundle cytokine family and a distant paralog of interleukin (IL)-7 that triggers dendritic cellmediated Thelper (Th)2 inflammatory responses [8]. Early studies showed that TSLP was expressed by human epithelial cells in the thymus, lung, intestine, skin, and stroma, as well as in tonsils and mast cells $[9,10]$. TSLP affects various cell types through a heterodimeric receptor consisting of the IL-7 receptor chain (IL-7R $\alpha$ ) and a specific subunit, TSLPR [11]. Overexpression of TSLP was detected in airway epithelial and mast cells of asthmatic patients and in mice with asthma, and TSLP overexpression correlated with both the expression of Th2-attracting chemokines and disease severity $[12,13]$. TSLP strongly activates dendritic cells freshly purified from the peripheral blood, then upregulates OX40L expression, which then interacts with OX40 on T cells, resulting in the production of Th2-attracting chemokines such as IL-4 and IL-13, and TNF- $\alpha$ [12,14]. The local application of an anti-TSLPR antibody $(\mathrm{Ab})$ and an anti-TSLP Ab prevented Th2-mediated airway inflammation [15,16]. AMG 157 is a human anti-TSLP monoclonal immunoglobulin G2 $\lambda$ that binds human TSLP and attenuates airway inflammation and the early and late asthmatic responses in patients with allergic asthma [17]. Thus, TSLP plays an important role in the context of allergic asthma. However, the mechanism of TSLP-mediated regulation in dysfunction of airway epithelial 
adherens junctions E-cadherin in HDM-induced asthma is still unknown. So we infer that TSLP might also mediate E-cadherin signaling

In the present study, we focused on the effects of TSLP signaling blocking on the expression and distribution of E-cadherin in a chronic HDM-induced mouse model of asthma and define the mechanism responsible for this in normal human bronchial epithelial cells.

\section{Materials and methods}

\subsection{Animals and reagent}

Specific-pathogen-free (SPF) BALB/c mice (male, six-weeks old, 20-24 g) were purchased from Southern Medical University (Guangzhou, China). The mice were housed in a SPF environment (room temperature $24^{\circ} \mathrm{C}$, humidity range $40-70 \%, 12 \mathrm{~h}$ light/dark cycle). Sterilized water and food were provided ad libitum. All experiments were conducted under the guidelines outlined by the committee of Southern Medical University on the use and care of animals. Standard guidelines for laboratory animal care followed the Guide for the Care and Use of Laboratory Animals. HDM was purchased from ALK-Abello A/S (Denmark), methacholine was obtained from Sigma-Aldrich, anti-TSLP $\mathrm{mAb}$ was purchased from R\&D Systems (MAB555; USA), and recombinant human TSLP was obtained from R\&D systems.

\subsection{Animal experimental protocol}

BALB/c mice were randomly assigned to one of 4 groups: (1) control group, phosphate-buffered saline (PBS; Gibco, Life Technology); (2) anti-TSLP mAb group, treated intranasally with anti-TSLP $\mathrm{mAb}(20 \mu \mathrm{g} /$ mouse per day, dissolved in PBS); (3) HDM group, treated intranasally with HDM (400 U/mouse per day, dissolved in deionized water); and (4) anti-TSLP mAb + HDM group, pretreated with anti-TSLP mAb 60 min prior to the intranasal treatment with HDM. The protocol for establishing the mouse model of HDM-induced asthma was as follows: The mice were exposed to $10 \mu \mathrm{l}$ of all treatments under inhaled sevoflurane-anesthesia for five consecutive days, followed by two days of rest, for a total of 8 weeks. No exogenous adjuvant was offered at any time. The anti-TSLP mAb dose was determined as described previously [15].

\subsection{The measurement of airway hyper-reactivity (AHR)}

As previously described [18], The measurement of AHR was carried out using methacholine. $24 \mathrm{~h}$ after the last administration, lung resistance $(\mathrm{RL})$ was assessed to evaluate the AHR. Mice were placed in a barometric plethysmo-graphic chamber (Buxco Electronics, Inc., Troy, NY) and provoked with vehicle (normal saline), followed by increasing concentrations of methacholine (6.25, $12.5,25$, or $50 \mathrm{mg} / \mathrm{ml}$ ) via a nebulizer (Buxco Electronics, Inc.) for $3 \mathrm{~min}$. Readings were taken and the bronchopulmonary resistance was expressed as the RL values. After finishing the test, the mice were sacrificed with an overdose of sodium pentobarbitone (administered intraperitoneally at a concentration of $100 \mathrm{mg} / \mathrm{kg}$ body weight; Sigma, China).

\subsection{Enzyme-linked immunosorbent assay (ELISA)}

Blood samples were collected, stored for $2 \mathrm{~h}$ at room temperature, and then centrifuged (3000g, $20 \mathrm{~min}$ ). The supernatants were harvested and stored at $-80^{\circ} \mathrm{C}$. Total serum IgE was measured by
ELISA (eBioscience, San Diego, US) according to the manufacturer's instructions. Next, bronchoalveolar lavage fluid (BALF) samples were collected and centrifuged immediately (3000 rpm, $10 \mathrm{~min}$ ). The supernatants were harvested and stored at $-80^{\circ} \mathrm{C}$. Then, the levels of interferon (IFN)- $\gamma$, IL-4, IL-5, and TSLP in the processed BALF samples were measured using ELISA (eBioscience, San Diego, US) according to the manufacturer's instructions.

\subsection{Pathological staining and Immunohistochemistry}

The left lungs of mice were removed and embedded in paraffin. Lung sections $(4 \mu \mathrm{m})$ were prepared with a Leica microtome 2030 (Leica Microsystems Nussloch GmbH, Nussloch, Germany). Lung tissues were stained with hematoxylin and eosin (H\&E) for blinded histopathologic assessment. Immunohistochemistry for TSLP, E-cadherin, and $\beta$-catenin was performed with the anti-TSLP (Abcam), anti-E-cadherin (Santa Cruz), and anti- $\beta$-catenin (Santa Cruz) antibodies as described previously [18].

\subsection{Epithelial cell culture and treatment}

The 16HBE human bronchial epithelial cells (Shanghai Fuxiang Biological Technology Co. Ltd., ATCC, USA) were grown in a cell culture flask in RPMI 1640 medium (Gibco, Life Technology) supplemented with $10 \%$ fetal bovine serum (Gibco, Life Technology). The cells were then incubated in a humidified incubator at $37{ }^{\circ} \mathrm{C}$ with an atmosphere of $5 \% \mathrm{CO}_{2}$. At $80-90 \%$ confluence, the cells were treated with trypsin and plated at a density of $10^{4}-10^{5}$ cells $/ \mathrm{cm}^{2}$ for use in the experiments. The medium was changed to serum-free RPMI 1640 when the cells reached complete confluence, and $12 \mathrm{~h}$ after that, the cells were treated according to the experimental plan.

\subsection{Immunofluorescence}

The immunofluorescence of cultured cells was monitored as described in our previous study [19,20]. Primary antibodies against E-cadherin and the FITC (green)-linked anti-rabbit IgG were obtained from Santa Cruz Biotechnology, USA. 4',6-diamidino-2-p henylindole dihydrochloride (DAPI) was obtained from SigmaAldrich.

\subsection{Western blotting analysis}

The treated cells and the tissue lysates of right lungs were collected and boiled in standard SDS sample buffer and western blotting for detection of the following antigens: Antibodies against TSLP (Abcam), phosphorylated (p)-AKT(Thr 308; Cell signaling technology), t-AKT (Cell signaling technology), E-cadherin (Santa Cruz Biotechnology), and $\beta$-catenin (Santa Cruz Biotechnology). The immunoreactive bands were exposed to Odyssey ${ }^{\circledR} \mathrm{CLx}$ Imager for image capture.

\subsection{Statistical analysis}

Statistical analysis was performing using the SPSS (version 19.0) software package. The variables were expressed as mean \pm standard deviation. One-way ANOVA accompanied by Bonferroni's post hoc test for multiple comparisons were utilized to compare differences among groups. Values of $\mathrm{P}<0.05$ were considered statistically significant. 


\section{Results}

3.1. HDM increases the expression of TSLP through PI3K/Akt signaling pathway in $16 \mathrm{HBE}$ cells

Initially, the effects of HDM on the expression of TSLP protein of $16 \mathrm{HBE}$ cells were examined. The 16HBE cells stimulated with HDM for the indicated periods of time $(0,2,4,8$ and $12 \mathrm{~h})$ and for the indicated concentrations $(0,200,400,800$ and $1600 \mathrm{U} / \mathrm{ml})$. We then assessed the expression levels of TSLP proteins by western blot analysis. These results revealed HDM $(400 \mathrm{U} / \mathrm{ml})$ caused a significant increase in the protein expression levels of TSLP for $8 \mathrm{~h}$ (Fig. 1A and B). In our previous study, we found that the HDM significantly increased the expression of p-AKT in vivo [18]. In order to identify the role of PI3K/Akt pathway on TSLP release induced by the HDM. The 16HBE cells were respectively treated with HDM $(400 \mathrm{U} / \mathrm{ml}$ ) with or without LY294002 (the inhibitor of PI3K). The results showed that the HDM significantly induced the expression of p-AKT and TSLP increased, whereas, LY294002 partially inhibited these changes (Fig. 1C). These findings indicate that the PI3K/Akt pathway regulated the HDM-induced the expression of TSLP.

\subsection{HDM and TSLP induce the delocalization of E-cadherin in $16 \mathrm{HBE}$} cells

Then we found that the exposure of 16HBE cells to HDM $(400 \mathrm{U} / \mathrm{ml})$ and TSLP $(10 \mathrm{ng} / \mathrm{ml})$ resulted in the aberrant distribution and cleavage of E-cadherin (Fig. 2A and B) for $12 \mathrm{~h}$. Intriguingly, we also found that $\operatorname{TSLP}(20 \mathrm{ng} / \mathrm{ml})$ can induce more marked changes in the delocalization of E-cadherin (Fig. 2B). Moreover, pre-treatment with LY294002 also could inhibit the
HDM-induced E-cadherin dysfunction (Fig. 2A). These findings indicate that HDM and TSLP have the ability to impairs the distribution of E-cadherin of 16HBE cells and the PI3K/Akt pathway may play a important role in the process.

\subsection{Neutralization of TSLP alleviates AHR and airway inflammation in the mouse model of HDM-induced asthma}

To confirm that the HDM-induced asthmatic mice model was successfully established and to address the role of TSLP and neutralization of TSLP in the murine asthma model, we first evaluated AHR in the HDM-induced asthma model by assessing the RL values. Compared with the saline-treated control group, the mice stimulated with HDM exhibited significantly increased responses and sensitivity to inhaled methacholine $(12.5,25$ and $50 \mathrm{mg} / \mathrm{ml}$; $\mathrm{P}<0.05)$. The anti-TSLP $\mathrm{mAb}$ did not increase murine AHR by itself; but, the RL values were partly restored after pretreatment with the anti-TSLP mAb (Fig. 3A). At the same time, upon examination of lung tissue using histopathological methods, we found that HDM-sensitized mice displayed a heavy infiltration of inflammatory cells and epithelial hyperplasia, while treatment with the anti-TSLP $\mathrm{mAb}$ markedly mitigated inflammatory infiltration in the peribronchial regions (Fig. 3B). Then, we evaluated the expression of TSLP by ELISA and immunohistochemical staining. TSLP protein expression levels were upregulated in the HDM-exposed mice, whereas pretreatment with the anti-TSLP mAb reduced TSLP expression, as expected (Fig. $3 \mathrm{C}$ and D). These data suggest that our mouse model of HDM-induced asthma is appropriate for addressing the role of airway inflammation and that TSLP is upregulated and released upon induction of asthma. Furthermore, in this model neutralization of TSLP mitigated AHR and airway inflammation.
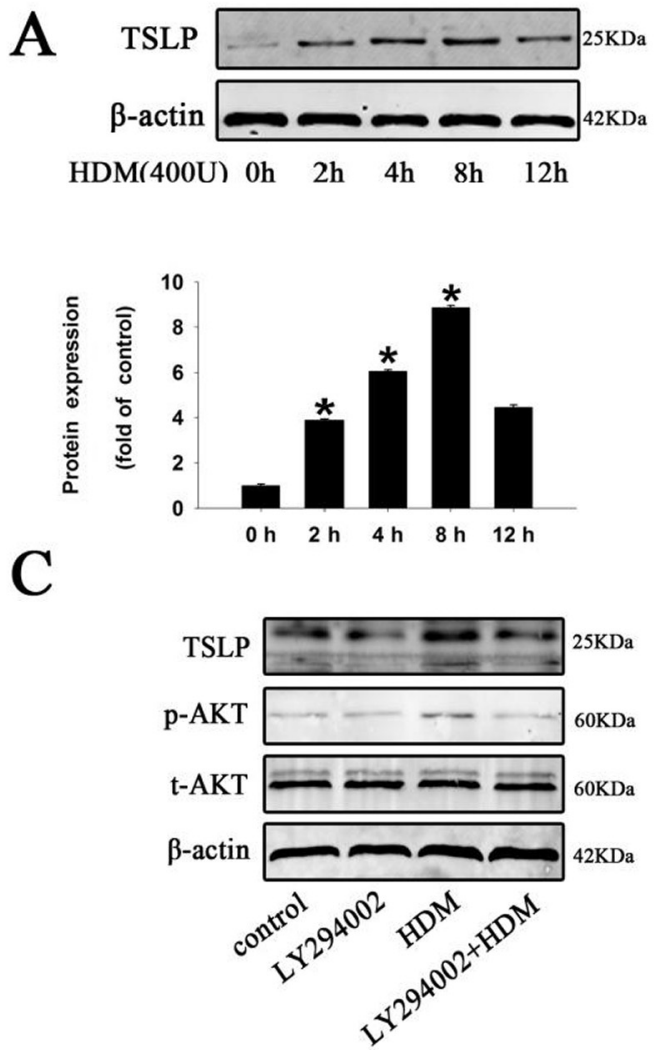

B

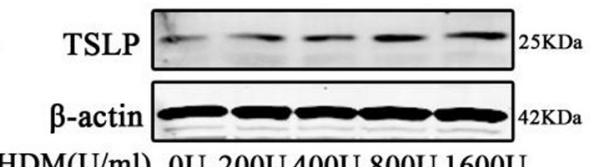

$\mathrm{HDM}(\mathrm{U} / \mathrm{ml})$ 0U 200U 400U 800U $1600 \mathrm{U}$
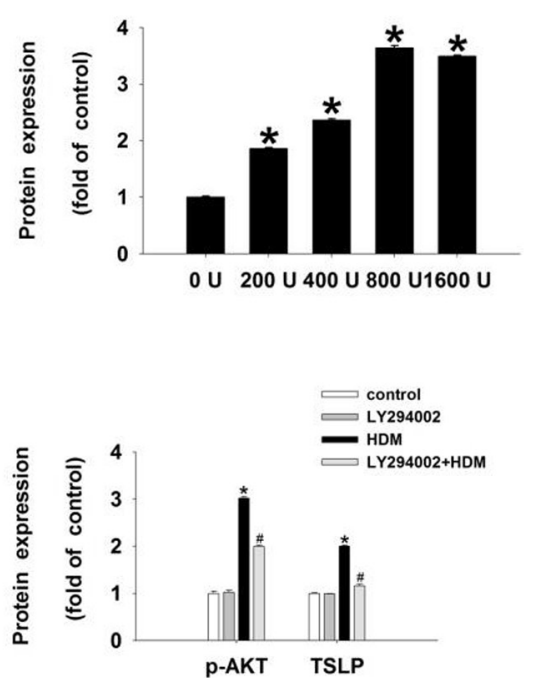

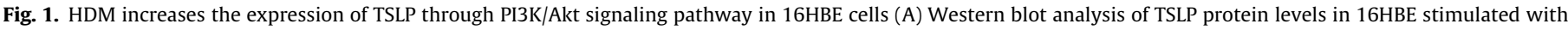

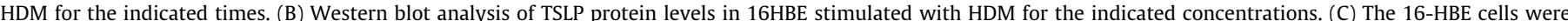

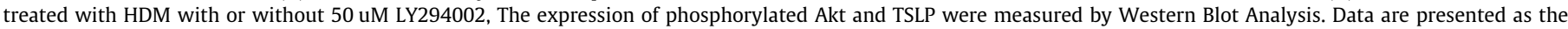
mean $\pm S D$. $n=3$. ${ }^{*} \mathrm{P}<0.05$ vs. the control group. ${ }^{\#} \mathrm{P}<0.05$, vs. the HDM group 
A

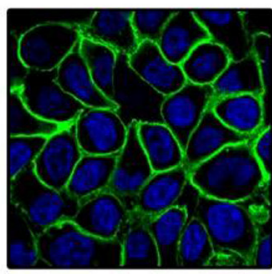

control

\section{E-cadherin}

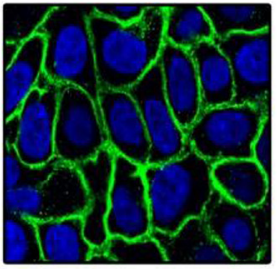

LY294002

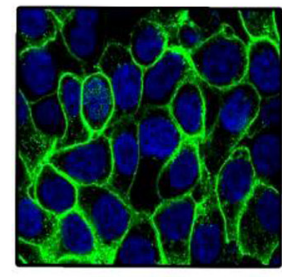

HDM

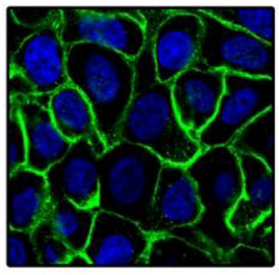

LY294002+HDM

B

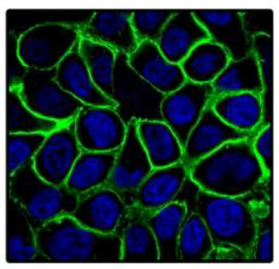

control

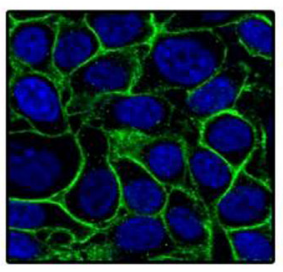

TSLP(10ng/ml)

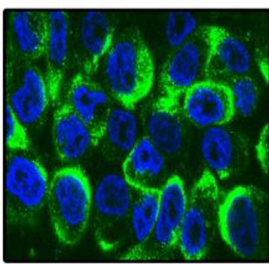

$\operatorname{TSLP}(20 \mathrm{ng} / \mathrm{ml})$

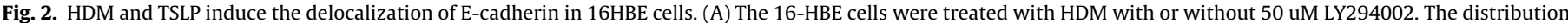

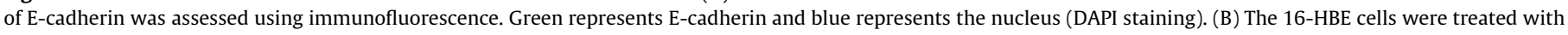

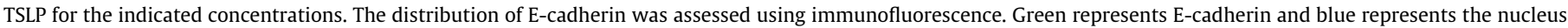
(DAPI staining). $\mathrm{n}=3$.
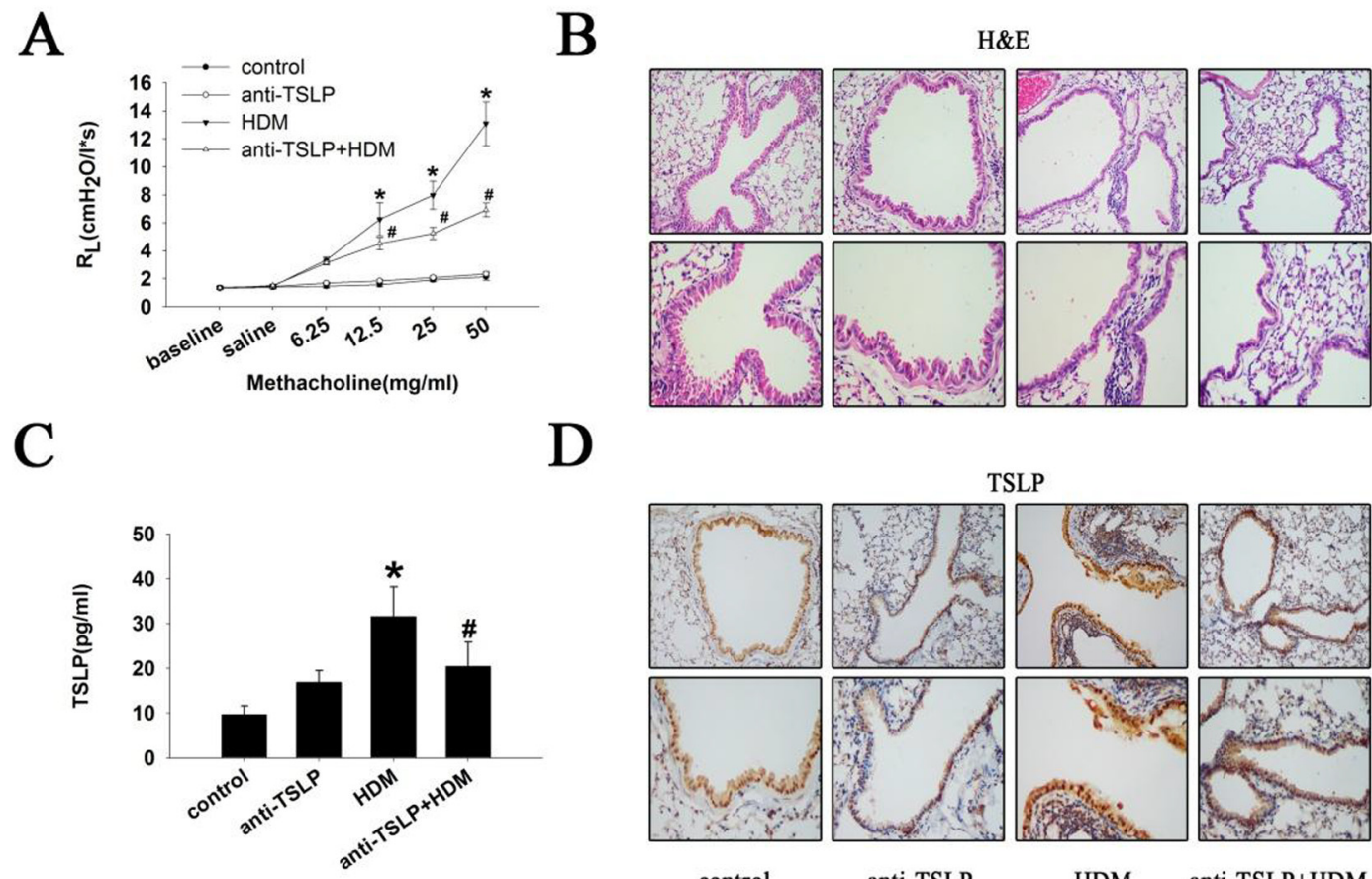

D

TSLP
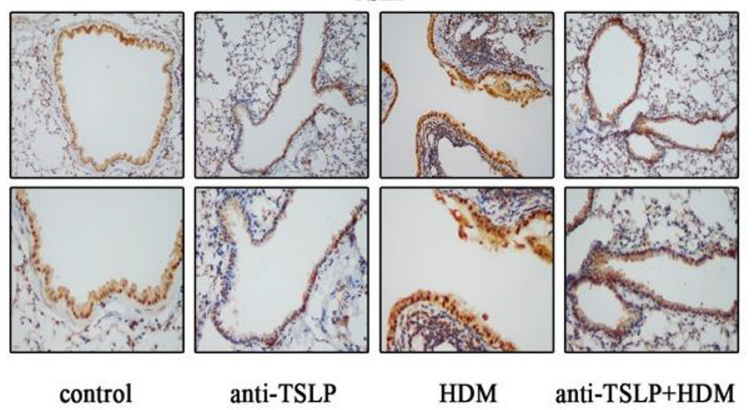

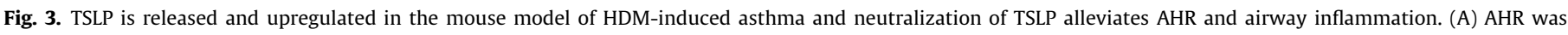

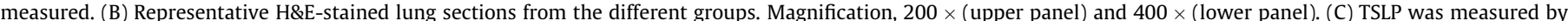

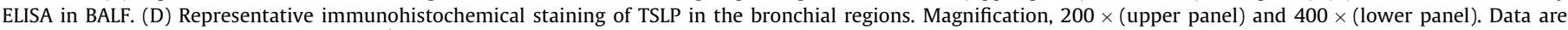
presented as the mean $\pm S D ; n=5 /$ group; ${ }^{*} \mathrm{P}<0.05$, vs. the control group; ${ }^{\#} \mathrm{P}<0.05$, vs. the HDM group.

\subsection{Neutralization of TSLP decreases the levels of serum IgE and} suppresses the release of Th2 cytokines in the mouse model of HDMinduced asthma

We analyzed the effect of TSLP neutralization on other parameters characteristic of airway inflammation in the mouse model of asthma. We found that the level of total serum IgE was markedly raised when the mice were sensitized with HDM, and that this effect was precluded by pretreatment with the anti-TSLP mAb (Fig. 4A). Then, we analyzed the levels of the Th1 cytokine IFN- $\gamma$ and the classical Th2-associated cytokines IL-4 and IL-5 in the BALF to elucidate the contribution of anti-TSLP treatment to Th1/Th2 

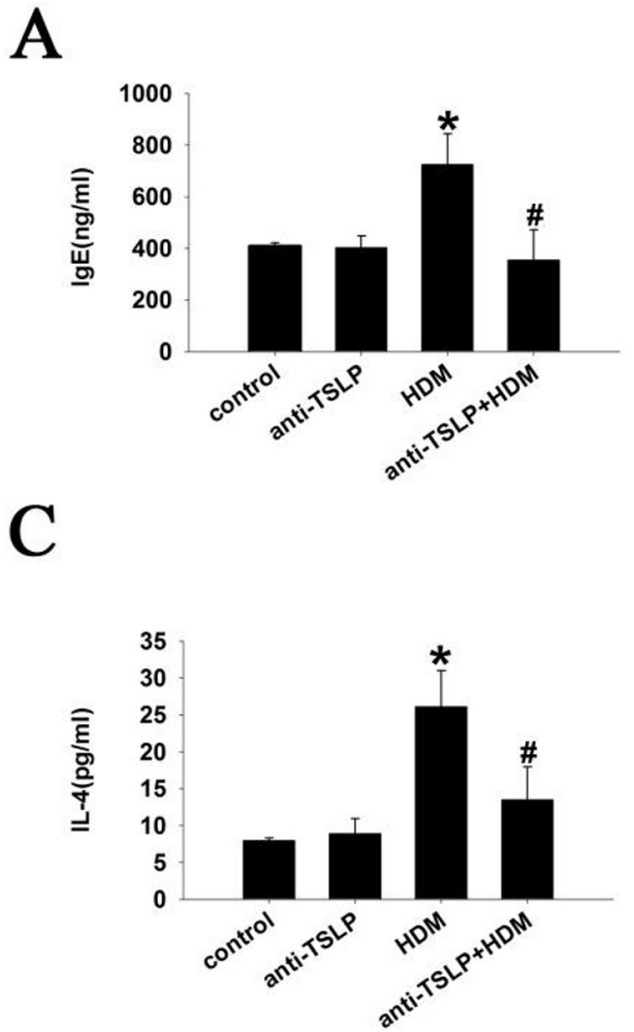

B

D
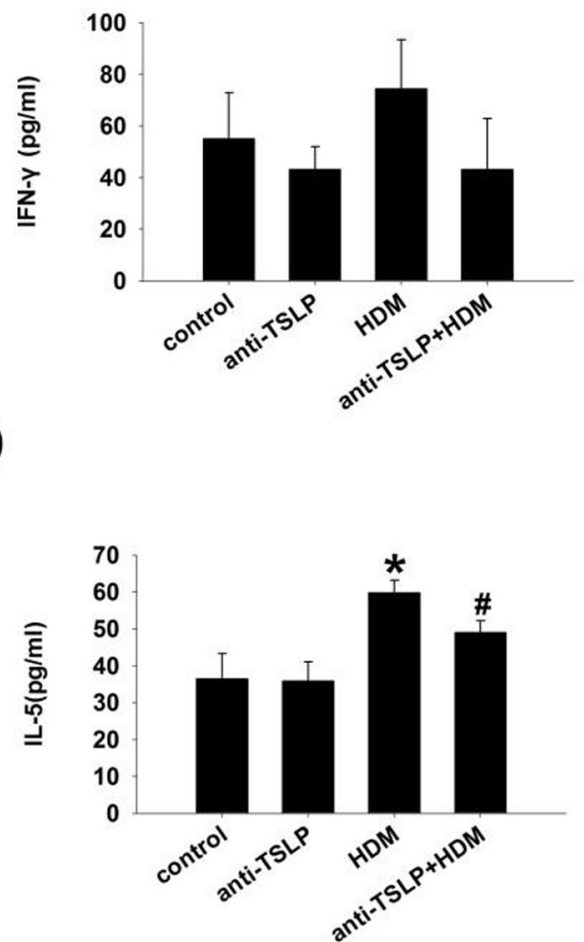

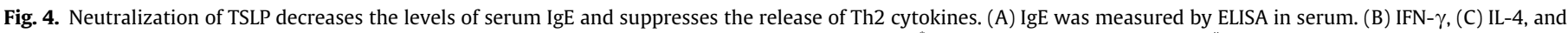
(D) IL-5 levels in BALF were measured by ELISA. Data are presented as the mean $\pm \mathrm{SD} ; \mathrm{n}=5 /$ group; ${ }^{*} \mathrm{P}<0.05$, vs. the control group; ${ }^{\#} \mathrm{P}<0.05$, vs. the HDM group.

cytokine immunoregulation in this mouse model. The HDMinduced release of IL-4 and IL-5 (Th2-related), but not IFN- $\gamma$ (Th1-related), was also suppressed by treatment with the antiTSLP mAb (Fig. 4B-D). These findings clearly indicate that exposure to HDM results in a robust Th2-driven inflammation in mice, and the protective effect of the anti-TSLP mAb, that of markedly suppressing the dominant production of Th2-associated cytokines and disruption of the Th1/Th2 balance inflammation, in our asthmatic mouse model.

3.5. Neutralization of TSLP inhibits the disruption and delocalization of E-cadherin and $\beta$-catenin by reducing the expression of phosphorylated $A K T$

E-cadherin and $\beta$-catenin mainly localized at the lateral side and apicolateral border of the airway epithelial cells in unstimulated cells, while exposure to HDM resulted in a notable decrease in E-cadherin and $\beta$-catenin at the epithelial cell-cell contact regions and a diffused staining pattern in the cytoplasm (Fig. 5A and B). These effects were partially rescued by treatment with the anti-TSLP mAb (Fig. 5A and B). Our results also showed a remarkable decrease of E-cadherin and $\beta$-catenin protein expression in HDM-treated group, whereas the anti-TSLP mAb protected against this HDM-induced decrease in both proteins, as indicated in the western blotting analysis (Fig. 5C). Therefore, we determined the effect of HDM and TSLP neutralization on the expression of p-AKT in lung tissue lysates from mice using western blotting. There was an increase in the ratio of p-AKT/t-AKT in the mouse model of HDM-induced asthma, and this increased phosphorylation AKT was significantly inhibited by pretreatment with the anti-TSLP mAb (Fig. 5C).

\section{Discussion}

In this study, we showed that HDM resulted in a increase in the protein expression levels of TSLP and E-cadherin dysfunction through PI3K/Akt signaling pathways in vitro. TSLP not only stimulated the redistribution of E-cadherin, but also played a pivotal role in mice with chronic HDM-induced asthma. For the first time, we demonstrated that neutralization of TSLP with an anti-TSLP $\mathrm{mAb}$ reduced airway hyperresponsiveness (AHR), airway inflammation, the levels of Th2-associated cytokines, including IL-4 and IL-5, and prevented the loss and redistribution of E-cadherin and $\beta$-catenin in the HDM-induced asthmatic mice through suppressing the phosphorylation of AKT signaling pathways.

Allergic asthma is characterized by airway epithelial barrier dysfunction, Th2-mediated airway inflammation, airway remodeling and AHR [1]. During this process, the integrity of the airway epithelial barrier is dependent on cellular integrity and strong cell-cell adhesion mediated by particular junctions [21]. Specifically, E-cadherin cooperates with $\beta$-catenin to form adherens junctions (AJs) and E-cadherin plays a crucial role in AJs as well as in apical tight junctions (TJs), providing the architectural support required for forming these junctional complexes [6,22]. Expression of E-cadherin is reduced in bronchial epithelium from asthma patients, compared with healthy persons $[4,6]$. It is well accepted that the loss of E-cadherin results in $\beta$-catenin transfer to the nucleus and its transcriptional activity and mediates activation of NF-kB-mediated transcription [23,24]. In this study, we showed that HDM exposure causes distribution anomalies of E-cadherin in $16 \mathrm{HBE}$ cells. We also established a chronic asthmatic mouse model successfully using prolonged intranasal administration of HDM. Our HDM-induced asthmatic mouse model displayed similar characteristics as those seen in our previous study [18]: AHR, a 
A

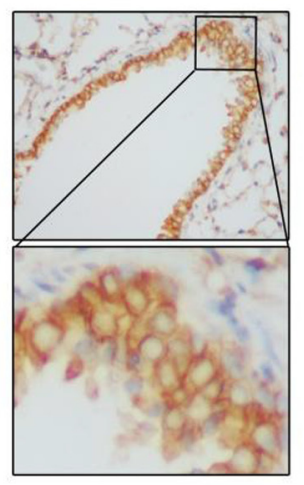

B

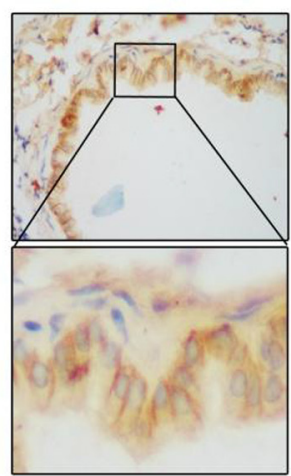

control
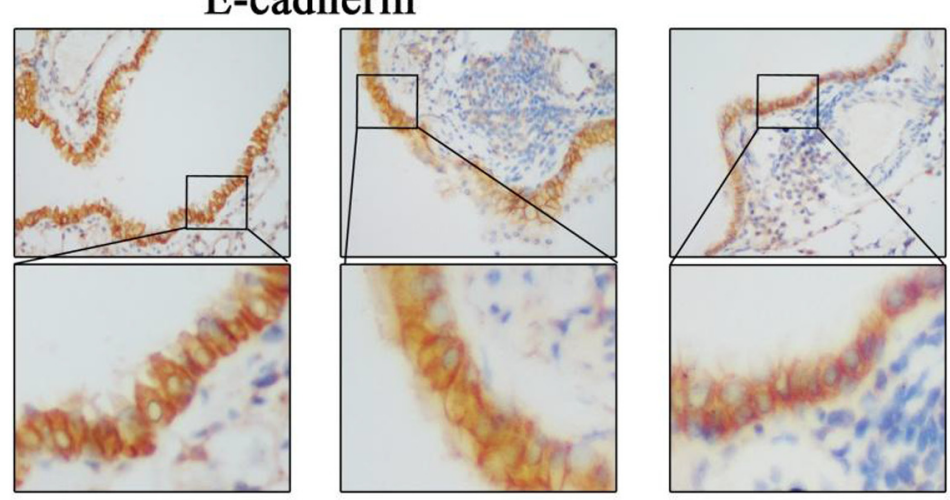

$\beta$-catenin

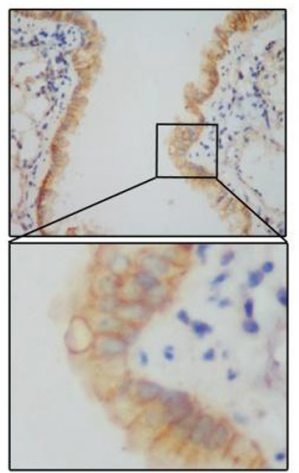

anti-TSLP

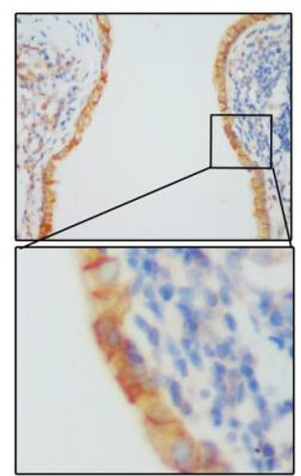

HDM

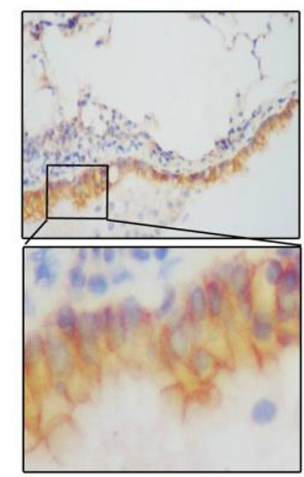

anti-TSLP+HDM

C
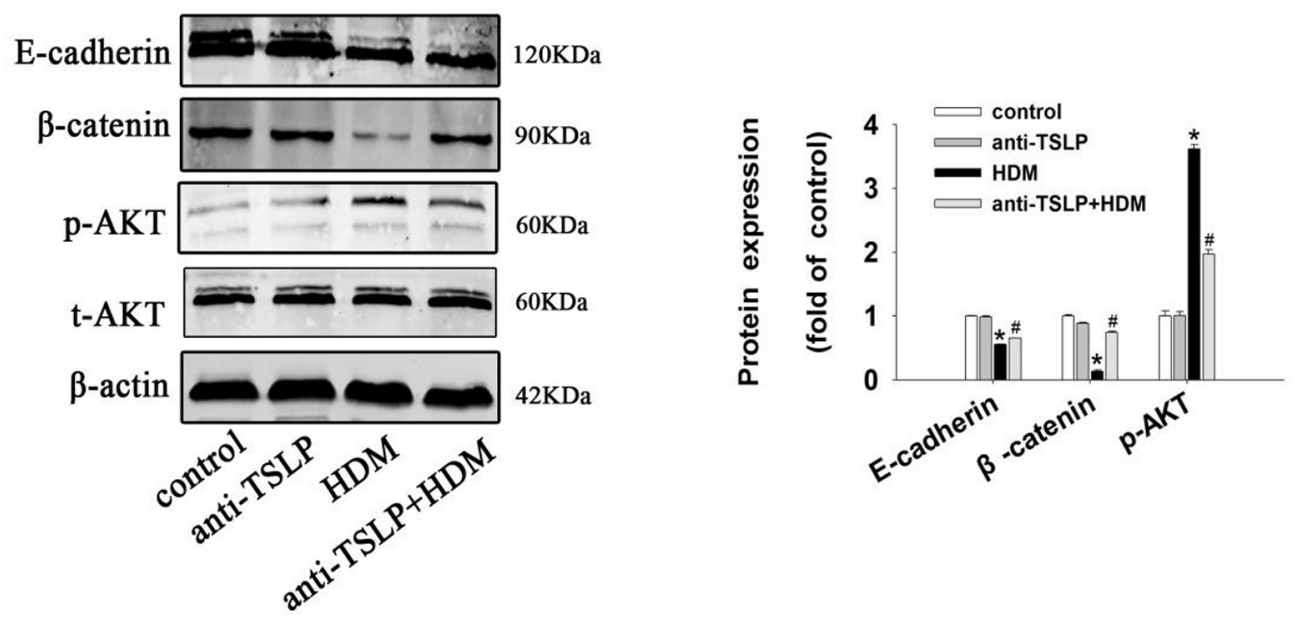

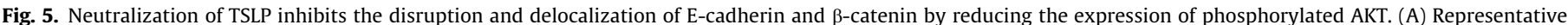

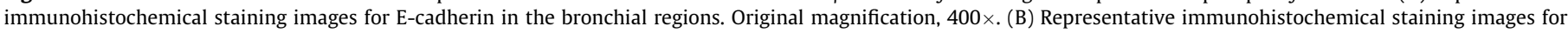

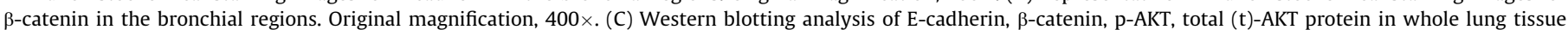

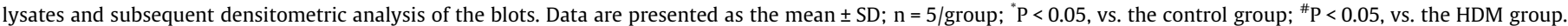

heavy infiltration of inflammatory cells, higher levels of serum IgE, Th2-dominated airway inflammation and the loss and redistribution of E-cadherin and $\beta$-catenin. The above results suggested that the disruptions of E-cadherin and $\beta$-catenin play an important role in HDM-induced asthma in mice and $16 \mathrm{HBE}$ cells. We did not further investigate the possible relationship between E-cadherin and $\beta$-catenin, an area we intend to explore in future studies.
Extensive studies have focused on the role of TSLP in allergic inflammatory responses [25]. As a cytokine that is highly expressed by the epithelium, TSLP is produced primarily by damaged epithelial cells and activate dendritic cells, and promotes differentiation of $\mathrm{CD}^{+} \mathrm{T}$ cells into pro-inflammatory $\mathrm{TH} 2$ cells that release IL-4, IL-5, IL-13, and TNF- $\alpha$ [26]. When a human airway epithelial cell line (BEAS-2B) or normal human bronchial epithelial 
cells were exposed to Toll-like receptor agonists and protease(s), TSLP was potently induced $[27,28]$. The elevated expression of TSLP in airways of patients with asthma leads to elevated expression of Th2, but not Th1, thus attracting chemokines [12]. TSLP expression is upregulated in Ovalbumin (OVA)-induced murine models of asthma [13]. Consistent with data from these previous studies, we showed that TSLP expression was significantly increased in the exposure of $16 \mathrm{HBE}$ cells to HDM and in the mouse model of HDM-induced asthma, suggesting the possible involvement of TSLP in the development of airway inflammation and disruptions in E-cadherin and $\beta$-catenin function. Specifically, TSLP can directly act on both mouse and human $\mathrm{CD}^{+}$and contributes to proliferation of $T$-cell lymphopoiesis [29,30]. In bronchial epithelial cells, TSLP is a homeostatic cytokine that regulates proliferation and injury repair [31]. TSLP promotes differentiation of fibroblasts to myofibroblasts and plays an important role in asthmatic airway remodeling [32]. We have found that HDM exposure causes increasing of TSLP protein expression in mice and 16HBE cells. However, the functional role of TSLP in E-cadherin dysfunction of airway epithelium is still unclear. In this study, we demonstrated that TSLP promotes the dislocation of E-cadherin in 16HBE cells to a greater extent than that induced by HDM. These data support the conclusion that TSLP may be a key factor in HDM-induced E-cadherin dysfunction.

To further address the roles of TSLP in the HDM-induced asthmatic mouse model, we investigated the effect of TSLP neutralization with an anti-TSLP mAb. In a previous study, local application of anti-TSLPR was shown to prevent Th2-mediated airway inflammation in OVA-primed asthmatic mice [16] and TSLP receptor (TSLPR)-deficient $\left(\mathrm{Tslpr}^{-1-}\right.$ ) mice exhibited remarkably decreased airway inflammation upon OVA-challenge [33]. Moreover, local blockage of TSLP eliminated airway inflammation and airway structural remodeling in an HDM-induced mouse model [15] and attenuated airway inflammation in patients with allergic asthma [17]. Consistent with these data, the blockade of TSLP with antiTSLP $\mathrm{mAb}$ reduced HDM-induced changes in our mouse asthma model as well. The disruption of E-cadherin and airway inflammation influence each other, which then contributes to the pathogenesis and exacerbation of asthma. Our data indicate that pretreatment with a high-affinity potent anti-TSLP mAb partly reduced AHR from methacholine inhalation and alleviated the concomitant heavy infiltration of inflammatory cells and epithelial hyperplasia in mice with HDM-induced asthma. Furthermore, we found that chronic HDM-exposure stimulated a robust Th2 response that was accompanied by more modest changes in IFN$\gamma$ levels in mice, suggesting a shift in the th $1 / 2$ balance toward Th2; this suggestion is supported by findings of our previous study [18]. As classical Th2 cytokines in asthma, IL-4 is important in the $\mathrm{CD}^{+} \mathrm{T}$ cell commitment to a Th2 phenotype, whereas IL-5 regulates eosinophil development and IgE has a positive correlation with eosinophil inflammation [26]. However, pretreatment with the anti-TSLP mAb dramatically reduced serum IgE levels and HDM-induced Th2 responses by decreasing the levels of Th2associated cytokines, IL-4 and IL-5. Simultaneously, we also found that overexpression of TSLP was accompanied by disruptions in expression and function of E-cadherin and $\beta$-catenin. Moreover, the anti-TSLP mAb partly restored the expression and dislocation of E-cadherin and $\beta$-catenin in mice. These data indicate that TSLP participates in promoting the release of Th2 cytokines and E-cadherin dysfunction in an animal model of HDM-induced asthma and that the anti-TSLP mAb counteracts the effects of TSLP and alleviates HDM-induced changes in the mouse asthma model. Further studies are warranted to delineate the molecular mechanisms underlying the protective effects of the anti-TSLP mAb and its utility in clinical asthma management.
We have previously reported that the HDM significantly induce the expression of p-AKT and the VEGF mediated endothelial barrier regulation by the PI3K/Akt pathway $[18,34]$. In our experiment, the HDM could induce the upregulation of TSLP, the phosphorylated Akt expression and the disruptions of E-cadherin in 16HBE cells, and pre-treatment with LY294002 (the inhibitor of PI3K) could inhibit the HDM-induced upregulation of TSLP and E-cadherin dysfunction. These data show that there are interaction relationship between the upregulation of TSLP and activation of the PI3K/Akt signaling pathway in regulation of HDM-induced E-cadherin dysfunction. Meanwhile we confirmed that the phosphorylation of AKT were elevated in the mouse model of HDM-induced asthma. Interestingly, the anti-TSLP mAb exerted suppressive effects on AKT activation, indicating that the inhibition of the phosphorylation of Akt may be responsible, at least in part, for the protective effects of the anti-TSLP mAb.

Taken together, our results demonstrate that HDM exposure promote the upregulation of TSLP and E-cadherin disruption by the PI3K/Akt pathway. The local application of an anti-TSLP mAb prevents HDM-induced airway epithelial barrier disruption and airway inflammation in mice, and the inhibition of the AKT pathways may be involved in this process. These findings provide additional insights into the importance of TSLP in asthma and TSLP signaling blocking can be as a potential therapeutic target.

\section{Conflicts of interest}

All authors declare no conflicts of interest associated with this manuscript.

\section{Acknowledgments}

This study was funded by the National Natural Science Foundation of China [81470228, 81270087, 81500023, 81300029, $81270089,81670026]$, the Precision Medicine Research of The National Key Research and Development Plan of China [2016YFC0905800], the National Program on Key Basic Research Project of China [973 Program, 2012CB518203], and Natural Science Foundation of Guangdong Province [S2013040013505, 2014A030310325, 2015A030313236] Scientific and Technological Project of Guangdong Province [2016A020215117] and Medical Research Foundation of Guangdong Province [201512223175676].

\section{References}

[1] B.N. Lambrecht, H. Hammad, The immunology of asthma, Nat. Immunol. 16 (2014) 45-56.

[2] C. Xiao, S.M. Puddicombe, S. Field, J. Haywood, V. Broughton-Head, et al., Defective epithelial barrier function in asthma, J. Allergy Clin. Immunol. 128 (2011), 549-556.e12.

[3] B.N. Lambrecht, H. Hammad, The airway epithelium in asthma, Nat. Med. 18 (2012) 684-692.

[4] I.H. Heijink, P.M. Kies, H.F. Kauffman, D.S. Postma, A.J.M. van Oosterhout, E. Vellenga, Down-regulation of E-cadherin in human bronchial epithelial cells leads to epidermal growth factor receptor-dependent Th2 cell-promoting activity, J. Immunol. 178 (2007) 7678-7685.

[5] J.A. Tunggal, I. Helfrich, A. Schmitz, H. Schwarz, D. Gunzel, M. Fromm, R. Kemler, T. Krieg, C.M. Niessen, E-cadherin is essential for in vivo epidermal barrier function by regulating tight junctions, EMBO J. 24 (2005) 1146-1156.

[6] M.C. Nawijn, T.L. Hackett, D.S. Postma, A.J.M. van Oosterhout, I.H. Heijink, E-cadherin: gatekeeper of airway mucosa and allergic sensitization, Trends Immunol. 32 (2011) 248-255.

[7] S. Post, M.C. Nawijn, T.L. Hackett, et al., The composition of house dust mite is critical for mucosal barrier dysfunction and allergic sensitisation, Thorax 67 (2012) 488-495

[8] S.F. Ziegler, F. Roan, B.D. Bell, et al., The biology of thymic stromal lymphopoietin (TSLP), Adv. Pharmacol. (San Diego, Calif.) 66 (2013) 129-155.

[9] P. Moon, H. Kim, Thymic stromal lymphopoietin is expressed and produced by caspase-1/NF- $\kappa$ B pathway in mast cells, Cytokine 54 (2011) 239-243.

[10] S.T. Holgate, The epithelium takes centre stage in asthma and atopic dermatitis, Trends Immunol. 28 (6) (2007). 
[11] P. A, O. K, B. H, et al., Cloning of a receptor subunit required for signaling by thymic stromal lymphopoietin, Nat. Immunol. 1 (1) (2000).

[12] S. Ying, B. O'Connor, J. Ratoff, Q. Meng, et al., Thymic stromal lymphopoietin expression is increased in asthmatic airways and correlates with expression of TH2-attracting chemokines and disease severity, J. Immunol. 174 (2005) 8183-8190.

[13] B. Zhou, M.R. Comeau, T.D. Smedt, et al., Thymic stromal lymphopoietin as a key initiator of allergic airway inflammation in mice, Nat. Immunol. 6 (2005) 1047-1053.

[14] T. Ito, Y. Wang, O. Duramad, et al., TSLP-activated dendritic cells induce an inflammatory Thelper type 2 cell response through OX40 ligand, J. Exp. Med. 202 (2005) 1213-1223.

[15] Z. Chen, T. Zhang, H. Li, et al., Neutralization of TSLP Inhibits Airway Remodeling in a Murine Model of Allergic Asthma Induced by Chronic Exposure to House Dust Mite, PLoS one 8 (2013) e51268.

[16] L. Shi, S. Leu, F. Xu, et al., Local blockade of TSLP receptor alleviated allergic disease by regulating airway dendritic cells, Clin. Immunol. 129 (2008) $202-$ 210.

[17] G.M. Gauvreau, P.M. O’Byrne, L. Boulet, et al., Effects of an Anti-TSLP Antibody on Allergen-Induced Asthmatic Responses, N. Engl. J. Med. 370 (2014) 21022110.

[18] C. Huang, H. Dong, M. Zou, et al., Bevacizumab reduced auto-phosphorylation of VEGFR2 to protect HDM-induced asthma mice, Biochem. Biophys. Res. Commun. 478 (2016) 181-186.

[19] W. Li, H. Dong, H. Zhao, et al., 1,25-Dihydroxyvitamin D3 prevents toluene diisocyanate-induced airway epithelial barrier disruption, Int. J. Mol. Med. (2015).

[20] R. Zhang, H. Zhao, H. Dong, et al., 1 2,25 -Dihydroxyvitamin D3 counteracts the effects of cigarette smoke in airway epithelial cells, Cell. Immunol. 295 (2015) 137-143.

[21] S. Ganesan, A.T. Comstock, U.S. Sajjan, Barrier function of airway tract epithelium, Tissue Barriers 1 (2013) e24997.

[22] C.J. Vallorosi, K.C. Day, X. Zhao, et al. Truncation of the beta-catenin binding domain of E-cadherin precedes epithelial apoptosis during prostate and mammary involution, J. Biol. Chem. 275 (2000) 3328-3334.
[23] G. Solanas, M. Porta-de-la-Riva, C. Agusti, et al., E-cadherin controls betacatenin and NF-kappa B transcriptional activity in mesenchymal gene expression, J. Cell Sci. 121 (2008) 2224-2234.

[24] S. Orsulic, O. Huber, H. Aberle, S. Arnold, R. Kemler, E-cadherin binding prevents beta-catenin nuclear localization and beta-catenin/LEF-1-mediated transactivation, J. Cell Sci. 112 (Pt 8) (1999) 1237-1245.

[25] S.F. Ziegler, Thymic stromal lymphopoietin and allergic disease, J. Allergy Clin. Immunol. 130 (2012) 845-852.

[26] Y. Liu, Thymic stromal lymphopoietin: master switch for allergic inflammation, J. Exp. Med. 203 (2006) 269-273.

[27] R. Segawa, N. Hirasawa, Exacerbation of allergic diseases by chemicals: role of TSLP, J. Pharmacol. Sci. 124 (2014) 301-306.

[28] H. Kouzaki, S.M. O'Grady, C.B. Lawrence, et al., Proteases Induce Production of Thymic Stromal Lymphopoietin by Airway Epithelial Cells through ProteaseActivated Receptor-2, J. Immunol. 183 (2009) 1427-1434.

[29] Q. Wang, J. Du, J. Zhu, X. Yang, B. Zhou, Thymic stromal lymphopoietin signaling in $\mathrm{CD}^{+} \mathrm{T}$ cells is required for TH2 memory, J. Allergy Clin. Immunol. 135 (2015), 781-791.e3.

[30] Y. Rochman, M. Kashyap, G.W. Robinson, et al, Thymic stromal lymphopoietin-mediated STAT5 phosphorylation via kinases JAK1 and JAK2 reveals a key difference from IL-7-induced signaling, Proc. Natl. Acad. Sci. 107 (2010) 19455-19460

[31] A. Semlali, E. Jacques, L. Koussih, et al., Thymic stromal lymphopoietininduced human asthmatic airway epithelial cell proliferation through an IL13-dependent pathway, J. Allergy Clin. Immunol. 125 (2010) 844-850.

[32] J. Wu, F. Liu, J. Zhao, et al., Thymic stromal lymphopoietin promotes asthmatic airway remodelling in human lung fibroblast cells through STAT3 signalling pathway, Cell Biochem. Funct. 31 (2013) 496-503.

[33] A. Al-Shami, R. Spolski, J. Kelly, et al., A role for TSLP in the development of inflammation in an asthma model, J. Exp. Med. 202 (2005) 829-839.

[34] A. Pedram, M. Razandi, E.R. Levin, Deciphering vascular endothelial cell growth factor/vascular permeability factor signaling to vascular permeability. Inhibition by atrial natriuretic peptide, J. Biol. Chem. 277 (46) (2002) 4438544398. 